\title{
TAXA DE DOAÇÕES EFETIVAS NO BRASIL E CORRELAÇÃO COM O ÍNDICE DE DESENVOLVIMENTO HUMANO MUNICIPAL
}

\author{
Effective donation rates in Brazil and correlation with the municipal human \\ development index
}

\author{
Arlene Terezinha Cagol Badoch', Fábio Silveira², Altair Von Stein', Juliana Ribeiro Giugni,
}

\begin{abstract}
RESUMO
Introdução: O Sistema Nacional de Transplantes está inserido no SUS, refletindo as desigualdades regionais. A relação do índice de desenvolvimento humano municipal (IDHM) com a doação de órgãos é pouco conhecida. Objetivo: Analisar o IDHM com os doadores efetivos de órgãos no ano de 2019. Método: Análise do banco de dados da Central Estadual de Transplantes do Paraná e do Registro Brasileiro de Transplantes do ano de 2019. Resultados: O IDHM médio é de $0,749 \pm 0,04$. A média de doações efetivas foi de $13,01 \pm 12,21$ por milhão de população $(\mathrm{pmp})$. Os estados considerados

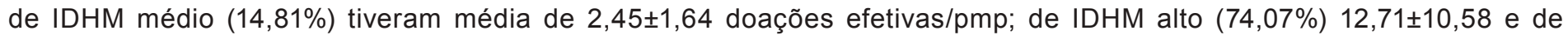
IDHM muito alto $(11,11 \%) 29,13 \pm 16,06$, com diferença significativa entre os grupos (p0,01). A correlação linear negativa é observada nos estados de IDHM médio e muito alto, positiva nos estados de IDHM alto. O número de doações efetivas/ pmp cresce $418 \%$ na transição da faixa de IDHM médio para alto e $129 \%$ na transição de IDHM alto para muito alto. Dentro dos subgrupos de IDHM há diferença significativa das taxas de doadores efetivos/pmp entre os estados (p0,000), denotando a existência de outros fatores que influenciam as taxas de doação. Santa Catarina e Paraná se destacam na gestão estadual do sistema. Conclusão: O IDHM se relaciona com as taxas de doadores de órgãos efetivos/pmp, porém, não é seu principal fator determinante.
\end{abstract}

Descritores: Obtenção de órgãos e tecidos; Transplante; Epidemiologia; Saúde Pública; Indicadores de Desenvolvimento.

Instituições:

${ }^{1}$ Central Estadual de Transplantes do Paraná. Curitiba, Paraná, Brasil.

2 Centro Digestivo e Transplante de Órgãos / Hospital do Rocio, Campo Largo, Paraná, Brasil.

\section{Correspondência:}

Fábio Silveira

E-mail: fabiosilveira@cdto.med.br

https://doi.org/10.53855/bjt.v24i2.010

Recebido em: 01/02/2021

Aceito em: 20/05/2021

\section{INTRODUÇÃO}

O Sistema Nacional de Transplantes (SNT) é o segundo maior programa de transplantes do mundo, sendo que quase a totalidade dos procedimentos é custeada pelo Sistema Único de Saúde. ${ }^{1}$

O processo de doação de órgãos é multíplice, envolve acesso ao tratamento, diagnóstico de morte encefálica, autorização familiar, manutenção do doador, mobilização das equipes multiprofissionais e realização do transplante. ${ }^{2} \mathrm{O}$ estado do Paraná tem destaque nacional ao lado de Santa Catarina na taxa de doadores de órgãos efetivos. ${ }^{3}$ 
A efetividade desse processo pode ser reflexo do desenvolvimento da sociedade, conforme analisado pelo Índice de Desenvolvimento Humano proposto pela Organização das Nações Unidas. ${ }^{4}$ Sua versão adaptada ao contexto nacional, o Índice de Desenvolvimento Humano Municipal (IDHM) tem sido utilizado como ponto de apoio para análises que envolvem longevidade, educação e renda da população brasileira. ${ }^{5,6}$

A análise de cada etapa do processo de doaçãotransplante pode ser capaz de auxiliar no planejamento de políticas públicas da área; dessa maneira, o conhecimento da influência do IDHM sobre esses índices nos estados brasileiros reveste-se de importância, sendo o objetivo do presente estudo.

\section{MÉTODOS}

Este estudo é uma análise do banco de dados da Central Estadual de Transplantes do Paraná e do Registro Brasileiro de Transplantes no ano de $2019,{ }^{3}$ analisando a taxa de doadores efetivos de órgãos para transplante.

A base populacional para os cálculos proporcionais foi obtida do Instituto Brasileiro de Geografia e Estatística. O IDHM e a estratificação de suas faixas - médio $(0,600$ $0,699)$, alto $(0,700-0,799)$ e muito alto $(0,800-1)$ - seguiu a definição do Atlas de Desenvolvimento Humano. ${ }^{6}$

Por definição, doador efetivo é um paciente com diagnóstico de morte encefálica, cuja família autorizou a doação e a cirurgia para captação de órgãos foi iniciada. ${ }^{7}$

Variáveis quantitativas foram avaliadas pela análise de variância (ANOVA) para dados paramétricos e teste de Kruskal-wallis para não paramétricos. A relação entre uma variável contínua e uma ou mais variáveis independentes foi realizada por regressão linear. A coleta e análises de dados foram realizadas pelo software Epidemiológico Epilnfo ${ }^{\mathrm{TM}}$ (versão 7.2.2.16, Center for Disease Control and Prevention). ${ }^{8}$ Testes estatísticos com significância, quando $p<0,05$.

Por utilizar somente dados agregados totalmente anônimos, o presente estudo dispensa necessidade de aprovação do Comitê de Ética em Pesquisa (CEP), conforme resolução $n^{\circ}$ 510/2016 do Conselho Nacional de Saúde (CNS).

\section{RESULTADOS}

O IDHM médio do Brasil foi de $0,749 \pm 0,04$, sendo o menor de Alagoas $(0,683)$ e o maior do Distrito Federal $(0,850)$. A média das doações efetivas no país foi de $13,01 \pm 12,21 \mathrm{pmp}$, sendo que o Amapá não apresentou doações e a maior taxa foi de 47,21 doações/pmp, em Santa Catarina (tabela 1).
Tabela 1 - IDHM e doações efetivas/pmp em cada estado brasileiro.

\begin{tabular}{|c|c|c|c|}
\hline Estado & IDHM & $\begin{array}{c}\text { Doações } \\
\text { efetivas/pmp }\end{array}$ & $\begin{array}{l}\text { Faixa do } \\
\text { IDHM }\end{array}$ \\
\hline Alagoas & 0,683 & 4,82 & Médio \\
\hline Maranhão & 0,687 & 1,42 & Médio \\
\hline Pará & 0,698 & 2,35 & Médio \\
\hline Piauí & 0,697 & 1,23 & Médio \\
\hline Acre & 0,719 & 5,75 & Alto \\
\hline Amapá & 0,74 & 0,00 & Alto \\
\hline Amazonas & 0,733 & 3,19 & Alto \\
\hline Bahia & 0,714 & 10,67 & Alto \\
\hline Ceará & 0,735 & 28,32 & Alto \\
\hline Espírito Santo & 0,772 & 11,33 & Alto \\
\hline Goiás & 0,769 & 10,84 & Alto \\
\hline Mato Grosso do Sul & 0,766 & 18,92 & Alto \\
\hline Mato Grosso & 0,774 & 0,87 & Alto \\
\hline Minas Gerais & 0,787 & 13,97 & Alto \\
\hline Paraíba & 0,722 & 5,50 & Alto \\
\hline Paraná & 0,792 & 43,79 & Alto \\
\hline Pernambuco & 0,727 & 19,48 & Alto \\
\hline Rio de Janeiro & 0,796 & 17,83 & Alto \\
\hline Rio Grande do Norte & 0,731 & 14,95 & Alto \\
\hline Rio Grande do Sul & 0,787 & 21,45 & Alto \\
\hline Rondônia & 0,725 & 13,66 & Alto \\
\hline Roraima & 0,752 & 3,47 & Alto \\
\hline Sergipe & 0,702 & 8,34 & Alto \\
\hline Tocantins & 0,743 & 1,93 & Alto \\
\hline Distrito Federal & 0,85 & 16,47 & Muito alto \\
\hline Santa Catarina & 0,808 & 47,21 & Muito alto \\
\hline São Paulo & 0,826 & 23,72 & Muito alto \\
\hline
\end{tabular}

Fonte: elaborado pelos autores (2020)

Os estados considerados de IDHM médio ( $n=4,14,81 \%)$ tiveram média de 2,45 $\pm 1,64$ doações efetivas/pmp; de IDHM alto $(n=20,74,07 \%) 12,71 \pm 10,58$ e de IDHM muito alto $(n=3,11,11 \%) 29,13 \pm 16,06$, com diferença significativa entre os grupos ( 0,01$)$.

O gráfico de dispersão demonstrando as correlações lineares do IDHM com a taxa de doadores efetivos/pmp está demonstrado na figura 1. Dentro dos subgrupos IDHM médio, alto e muito alto, há diferença significativa das taxas de doadores efetivos/pmp entre os estados (p0,000). A correlação linear negativa é observada nos estados de IDHM médio e muito alto e a correlação é positiva nos estados de IDHM alto. 
Figura 1 - Dispersão e correlação linear do IDHM com as doações efetivas/pmp.

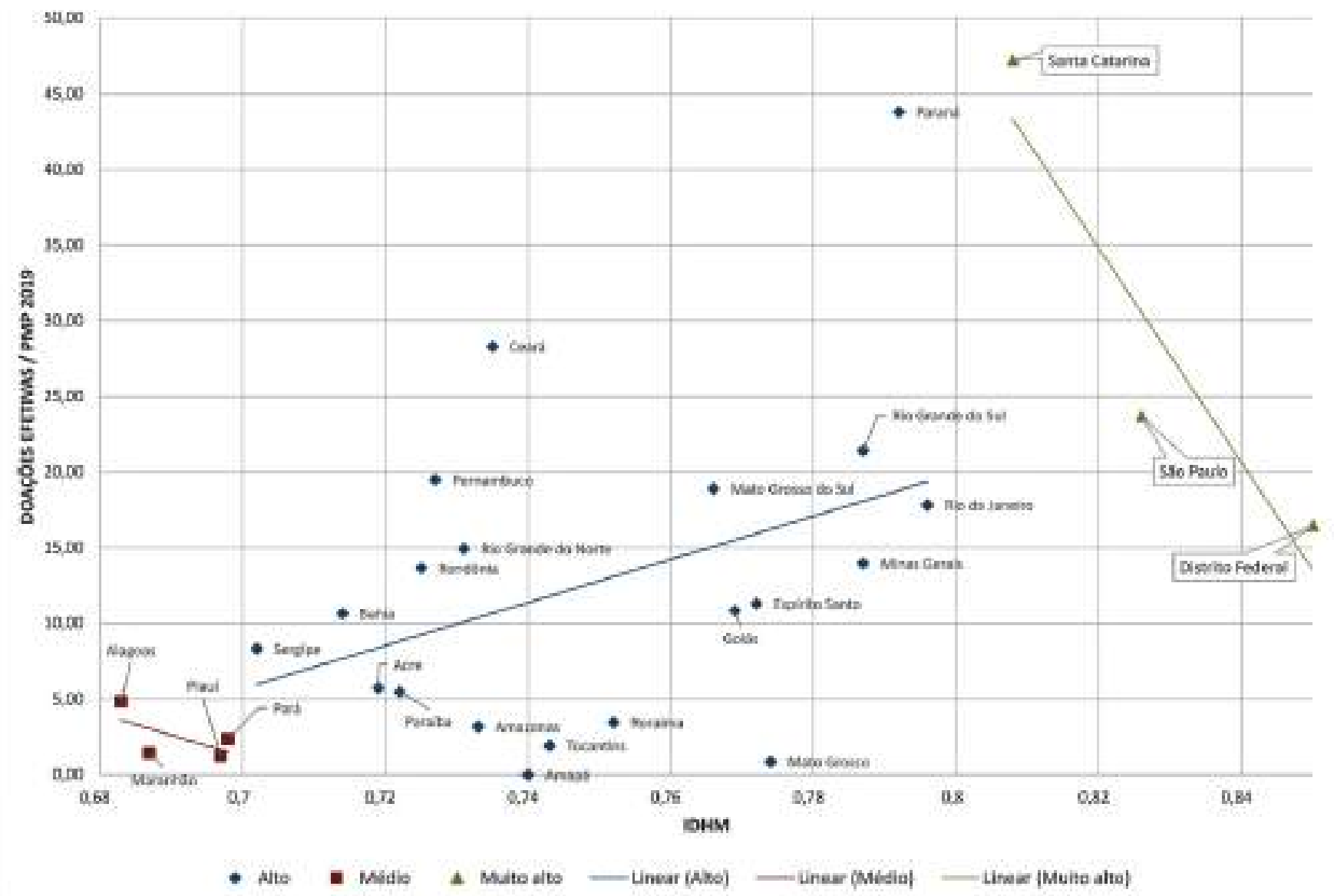

A variação percentual da taxa de doadores efetivos/pmp entre o melhor e o pior estado dentro da mesma faixa do IDHM é de $74 \%, 100 \%$ e $65,11 \%$, respectivamente, para a faixa de IDHM médio, alto e muito alto.
O número de doações efetivas/pmp cresce $418 \%$ na transição da faixa de IDHM médio para o alto e $129 \%$ na transição do IDHM alto para muito alto (figura 2).

Figura 2 - Crescimento das taxas de doação efetiva/pmp segundo faixa do IDHM

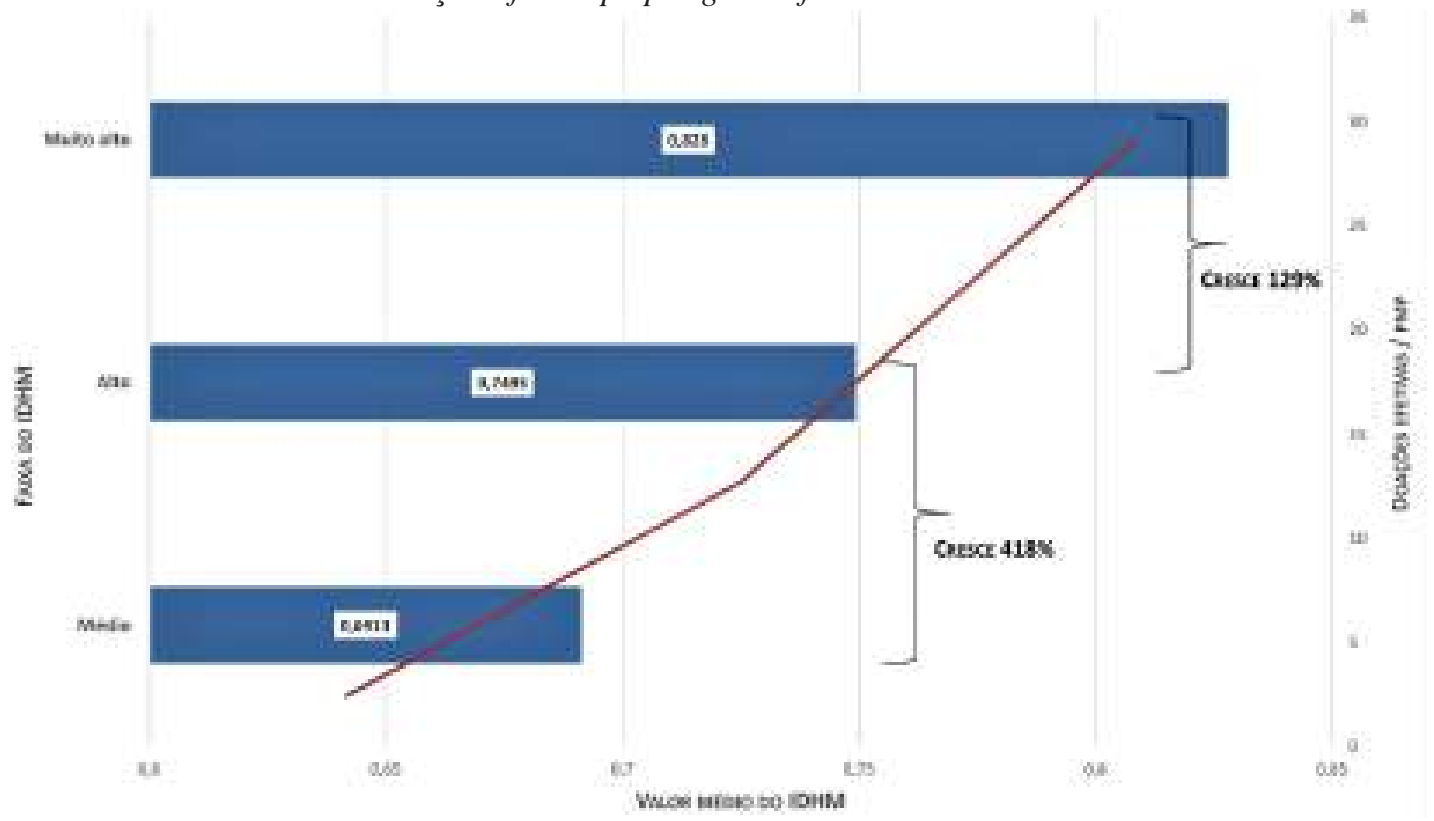




\section{DISCUSSÃO}

Um número crescente de doenças irreversíveis possui no transplante sua única possibilidade terapêutica. Essa necessidade de órgãos pressiona o sistema público de saúde - responsável pela organização do sistema de busca ativa - a aumentar sua eficiência, ${ }^{9} \mathrm{em}$ um cenário de constante racionamento de recursos financeiros e humanos. ${ }^{10}$

O Sistema Nacional de Transplantes está intrinsicamente ligado ao SUS, que reflete as desigualdades regionais de nosso país. ${ }^{11}$ A hierarquização do SUS nas esferas federal, estadual e municipal muitas vezes desnuda a diferença na efetividade da aplicação dos recursos públicos na área.

O IDHM é uma ferramenta de análise do desenvolvimento da sociedade, constituído de três componentes ${ }^{6}$ expectativa de vida, acesso à saúde e escolaridade - sendo que todos influenciam o processo de doaçãotransplante. Expectativa de vida porque a maior parte dos doadores é vítima de acidentes vasculares encefálicos, ${ }^{12}$ mais comuns após a quinta década de vida. Acesso à saúde porque uma doação efetiva é resultado de um atendimento médico estruturado, ${ }^{13}$ onde há relação entre o IDHM e a infraestrutura de saúde instalada. ${ }^{14}$ Escolaridade porque o nível de desenvolvimento intelectual influencia as taxas de negativas à de doação de órgãos. ${ }^{15}$

Observamos que há aumento das taxas de doação efetiva conforme o crescimento do IDHM (figura 2), o que reflete uma percepção prévia de que o índice refletiria melhor desenvolvimento de todas as etapas envolvidas no processo de doação. ${ }^{16}$ Não obstante, a correlação positiva entre esses índices não se repete ao estratificarmos pelas faixas do IDHM. Nos estados de IDHM alto, observamos correlação positiva, porém nos estados de IDHM médio e muito alto, a correlação é negativa (figura 1). Dito isso, as doações efetivas relacionam-se com o desenvolvimento da sociedade - aferido pelo IDHM - porém esse não é o único fator envolvido. ${ }^{1}$
A eficiência do sistema de procura de órgãos está muito relacionada ao sistema de busca ativa e da existência de aplicação dos protocolos de notificação e manutenção de morte encefálica, fatores esses de responsabilidade da esfera estadual, a cargo das Centrais Estaduais de Transplantes. Além disso, há influência da colaboração dos profissionais de saúde das unidades de terapia intensiva no processo de detecção e manutenção dos potenciais doadores. ${ }^{2}$

Esses fatores influenciadores relatados tornam-se evidentes ao constatarmos as diferenças significativas nas taxas de doadores efetivos/pmp entre estados pertencentes à mesma faixa de IDHM. Conhecendo o fato que o IDHM tem maior relevância sobre o desempenho do SUS, quando comparado ao percentual médio de investimento aplicado na saúde,${ }^{17}$ é evidente o fato que questões organizacionais são o grande fator diferencial na eficiência da gestão pública nessa área. A importância da gestão desnuda-se na observação da existência de correlação linear negativa nos estados de IDHM muito alto (figura 1).

A falta de órgãos para transplante não é resultado da falta de doadores, mas da falha de sua identificação, da obtenção de consentimento e da captação desses órgãos. ${ }^{12}$ É a gestão nas esferas estaduais - explicitadas nos exemplos dos estados do Paraná e Santa Catarina - que torna possíveis excelentes índices de doadores efetivos em relação às métricas internacionais, ${ }^{18,19}$ assim como quando comparados aos estados brasileiros de IDHM semelhantes.

\section{CONCLUSÃO}

O IDHM tem correlação com as taxas de doadores de órgãos efetivos/pmp no Brasil, porém outros fatores influenciam a métrica, como questões relativas ao sistema de busca ativa, manutenção e efetivação de doação dos potenciais doadores de órgãos. 


\section{ABSTRACT}

Introduction: The National Transplant System is inserted in the SUS, reflecting regional inequalities. The relationship between the municipal human development index (MHDI) and organ donation is unknown. Purpose: To analyze MHDI with effective organ donors in 2019. Methods. Analysis of the Transplant Coordination Center database in Paraná, and the Brazilian Registry of Transplants of the year 2019. Results: The mean MHDI is $0.749 \pm 0.04$. The effective donation average was $13.01 \pm 12.21$ per million population $(\mathrm{pmp})$. States considered as having average MHDI (14.81\%) had an effective donation average of $2.45 \pm 1.64$ / pmp; high MHDI (74.07\%) $12.71 \pm 10.58$ and very high MHDI (11.11\%) $29.13 \pm 16.06$, with significant difference between groups (p0.01). Negative linear correlation is observed in states of medium and very high MHDI, positive in states of high MHDI. The number of effective donations / pmp has grown $418 \%$ in the transition from medium to high MHDI range and $129 \%$ in the transition from high to very high MHDI. Within MHDI subgroups. there is a significant difference in the rates of effective donors / pmp between states $(\mathrm{p} 0,000)$, denoting the existence of other factors influencing the donation rates. Santa Catarina and Paraná stand out in the state management of the system. Conclusion: MHDI is related to the rates of effective organ donors / pmp, however it is not its main determining factor.

Keywords: Tissue and Organ Procurement; Transplant; Epidemiology; Public health. Development Indicators.

\section{REFERÊNCIAS}

1. Garcia VD, Abbud-Filho M, Felipe C, Pestana JM. An Overview of the Current Status of Organ Donation and Transplantation in Brazil. Transplantation. 2015 Aug;99(8):1535-7.Disponível em:http://dx.doi.org/10.1097/ TP.0000000000000828

2. Garcia VD. Por uma política de transplantes no Brasil. São Paulo: Office Editora; 2000. 165 p.

3. Registro Brasileiro de Transplantes. ABTO - Associaçao Brasileira de Transplante de Órgãos; 2019 [citado em 2020 oct 19]; 88]. Disponível em: https://site.abto.org.br/ publicacao/rbt-2019/.

4. Rahi M. Human development report 2010: Changes in parameters and perspectives. Indian J Public Health. 2011 Oct-Dec;55(4):272-5.Disponível em:http://dx.doi. org/10.4103/0019-557X.92404

5. HUMAN DEVELOPMENT REPORT 2019 Beyond income, beyond averages, beyond today:Inequalities in human development in the 21st century. United Nations Development Programme; 2019 [citado em 2020 09/28/2020]; Disponível em: http://hdr.undp.org/en/content/ human-development-index-hdi.

6. O Índice de Desenvolvimento Humano Municipal Brasileiro. Programa das Nações Unidas para o Desenvolvimento (PNUD), 2013. [citado em 2020 09/28/2020]; Disponível em: https://www.ipea.gov.br/portal/index.php?option=com_ content\&id=19153
7. EDQM. Guide to the safety and quality assurance for the transplantation of organs, tissues and cells. 4th ed. Strasbourg, France: European Comittee of Experts on Organ Transplantation; 2010.

8. Dean AG, Arner TG, Sunki GG, Friedman R, Lantinga $M$, Sangam $S$ et al. Epi Info ${ }^{\mathrm{TM}}$, a database and statistics program for public health professionals. CDC, Atlanta, GA, USA. 2011

9. Jox RJ, Assadi G, Marckmann G. Organ Transplantation in Times of Donor Shortage. 1a ed. Switzerland: Springer, Cham; 2016. 352 p.

10. Busuttil RW. Transplantation of the liver. Third ed: Elsevier Saunders; 2015.

11. Marinho A, Cardoso Sde S, Almeida VV. [Organ transplantation in Brazilian States: effectiveness, productivity, and capacity]. Cad Saude Publica. 2011 Aug;27(8):1560-8.Disponível em:http://dx.doi. org/10.1590/s0102-311x2011000800011

12. Matesanz R, Dominguez-Gil B. Strategies to optimize deceased organ donation. Transplantation Reviews. 2007 2007/10/01/;21(4):177-88.Disponível em:http:// dx.doi.org/https://doi.org/10.1016/j.trre.2007.07.005

13. Sheehy E, Conrad SL, Brigham LE, Luskin R, Weber $\mathrm{P}$, Eakin $\mathrm{M}$, et al. Estimating the number of potential organ donors in the United States. N Engl J Med. 2003 Aug 14;349(7):667-74. Disponível em:http://dx.doi. org/10.1056/NEJMsa021271 
14. Soares JJN, Machado MH, Alves CB. The Mais Medicos (More Doctors) Program, the infrastructure of Primary Health Units and the Municipal Human Development Index. Cien Saude Colet. 2016 Sep;21(9):2709-18.Disponível em:http:// dx.doi.org/10.1590/1413-81232015219.16432016

15. Bendorf A, Pussell BA, Kelly PJ, Kerridge IH. Socioeconomic, demographic and policy comparisons of living and deceased kidney transplantation rates across 53 countries. Nephrology (Carlton). 2013 Sep;18(9):633-40. Disponível em:http://dx.doi.org/10.1111/nep.12101

16. Mizraji R, Godino M, Tommasino N, Alvarez I. Donation rates: what matters? Transplant Proc. 2014 Nov;46(9):2940-4.Disponível em:http://dx.doi.org/10.1016/j. transproceed.2014.07.021
17. Mesquita MS. Influência do percentual aplicado na saúde e do Índice de Desenvolvimento Humano Municipal sobre o desempenho do SUS. Saúde Coletiva - Campus UnB Ceilândia: Universidade de Brasília; 2013.

18. Mizraji R, Alvarez I, Palacios RI, Fajardo C, Berrios C, Morales F, et al. Organ donation in Latin America. Transplant Proc. 2007 Mar;39(2):333-5.Disponível em:http://dx.doi.org/10.1016/j.transproceed.2007.01.017

19. Mahillo B, Carmona M, Alvarez M, Noel L, Matesanz R. Global Database on Donation and Transplantation: goals, methods and critical issues (www.transplantobservatory.org). Transplant Rev (Orlando). 2013 Apr;27(2):57-60.Disponível em:http://dx.doi.org/10.1016/j. trre.2013.01.001 